\title{
Influence of the main parameters of a parallel-plate dialyser under laminar flow conditions
}

\author{
Spas D Kolev ${ }^{1, *}$ and Willem E van der Linden \\ Laboratory for Chemical Analysus, Department of Chemical Technology, University of Twente, PO Box 217, \\ NL-7500 AE Enschede (Netherlands)
}

(Recerved 22nd Aprl 1991, revised manuscript received 14th October 1991)

\begin{abstract}
A mathematical model describing the mass transfer in a parallel-plate dialyser with co-current laminar flow in both channels based on the Navier-Stokes equations and Fick's second law was developed Numerical solutions are presented for pulse- and stepwise concentration changes of the solute in one of the channels ustng the Laplace transform technique By simulation the influence of the main design and operational parameters of the dialyser and the most important physical constants for the mass transfer process were investigated Conclusions with regard to optimum desıgn and operation were drawn and some possibılities for simplifying the model were established
\end{abstract}

Keywords Flow system, Dialysis, Mass transfer, Mathematical modelling

The proper understanding of the processes governing the heat and mass transfer in multstream flow systems is of primary importance in designing and operating a number of mass and heat exchange apparatuses finding wide application in industry [l], medıcine (e g , artıficıal kıdneys [2-6]) and chemical analysis (e g, on-line analytical dialysers in segmented [7] and unsegmented [8] continuous-flow analysis and in flowinjection analysis [9-12]) Their mathematical modelling has given a substantial impetus to the development of new techniques in the solution of the relevant partial differential equations which form an important class of equations in mathematical physics Some of these techniques have been successfully applied in the solution of mass transfer problems in other areas (e $\mathrm{g}$, mass transport in capıllary-tıssue systems $[13,14])$

\footnotetext{
1 Permanent address Faculty of Chemistry, University of Sofia, Anton Ivanov Ave 1, BG-1126 Sofia, Bulgana
}

Until the introduction of on-line dialysers in the manifolds for flow-injection analysis, the transient mass transfer in single- and multi-stream flow systems has been of limited practical interest because industrial heat and mass exchangers work under steady-state conditions and the overall effect of the transient periods (e g start-up, shutdown, power surge and pump fallure) is usually negligible The same is true for the haemodialysers used as artıficial kidneys If the time dependence of the mass transfer process is taken into consideration the problem becomes much more complicated from a mathematical point of view Probably this is one of the reasons why even membrane mass transfer in flow-injection manıfolds was modelled under steady-state conditions $[11,12]$ Only a small number of theoretical investigations have dealt with the dynamic heat or mass transfer in single-stream [15-18] and mult1stream [19] systems

The on-line analytical dialysers in flow-injection systems operate under dynamic conditions 
For the mathematical modelling of such flow-injection systems a proper understanding of the transient mass transfer in double-stream flow systems with a semipermeable membrane separating the two fluid phases is required The on-line flow-injection dialysers exhibit some important peculianties which distinguish them from the dialysers used in other areas First, neither the analyte concentration in the donor stream nor that in the acceptor stream can be considered as constant in space and time, as has been assumed in the mathematical modelling of haemodialysers Second, the thickness of the semipermeable membrane is usually of the same order of magnitude as the heights (diameters) of the donor and acceptor channels of the dialyser so that the mass transfer process within the membrane must be included in the overall mathematical description of the system Third, all mass-transfer processes of interest take place under dynamic conditions Among the various types of dialysers used in industry and in medical practice ( $\mathrm{e}$, parallelplate, tubular and spiral-plate dialysers), the parallel-plate dialysers have been used most frequently in flow-injection manifolds so far The Reynolds number corresponding to the flow-rate and the geometrical dimensions of these dialysers is well below the limit of turbulence so that laminar flow can be expected to prevall in them

The objective of this investigation was to elucidate the effect of the main parameters of a parallel-plate co-current dialyser under laminar flow conditions on the efficiency and to draw conclusions for improving its performance under dynamic and steady-state conditions

\section{MATHEMATICAL MODEL}

In the present investigation the dialyser is assumed to consist of three parallel plates The outer two are totally impermeable whereas the middle one separating the donor and the acceptor streams is permeable to the analyte A stepor pulse-function input at $x=0$ (Fig 1) is assumed to take place in the donor stream The

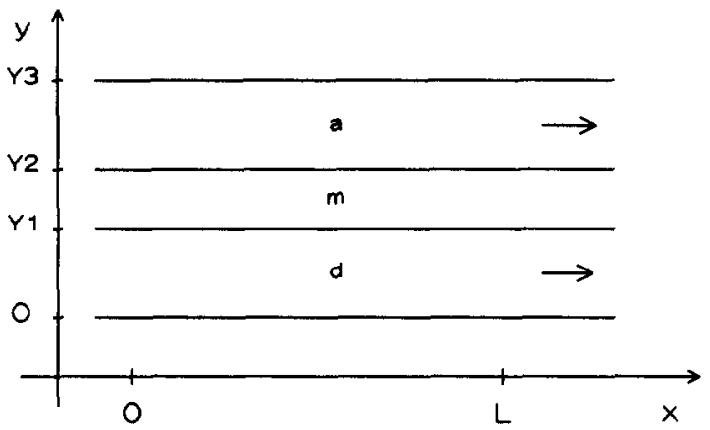

Fig 1 Scheme of the co-current flow system $Y 1=2 a_{\mathrm{d}}$, $Y 2=2\left(a_{\mathrm{d}}+\delta\right), Y 3=2\left(a_{\mathrm{d}}+\delta+a_{\mathrm{a}}\right), \mathrm{d}, \mathrm{m}$ and a refer to the donor stream, the membrane and the acceptor stream, respectively

other assumptions are the following the donor and the acceptor streams have constant physical properties, the flow in both channels is laminar, Newtonian and hydrodynamically fully developed, convection takes place only in the axial direction, the molecular diffusion coefficients for a particular solute in the donor and the acceptor stream and also in the membrane are constants independent of other solutes, the solute distribution coefficients for the donor stream and the membrane and for the acceptor stream and the membrane are constants which are not necessarly the same, or equal to unity, the transport of solute from the donor to the acceptor stream through the semipermeable membrane is caused by a concentration gradient only, the hydrostatic and osmotic pressure differences between the fluids are considered to be negligible and there is no convection through the membrane, diffusion in both channels and in the membrane follows Fick's second law, no sources or sinks exist in the system, and the concentrations of the solutes are low so that their activity coefficients can be assumed to be equal to unity

The mathematical model consists of two convective-diffusion equations for the case of parallel-plate lamınar flow and two-dımensıonal Fick's second law applied to the membrane In dimensionless quantities and variables these equations have the following form (the original equations 
TABLE 1

Symbols and definitıons a

\begin{tabular}{|c|c|}
\hline$a$ & Half of channel height (m) \\
\hline$A$ & $=a_{\mathrm{a}} / a_{\mathrm{d}}$ \\
\hline$c$ & Concentration $\left(\mathrm{mol} \mathrm{m}^{-3}\right)$ \\
\hline$\hat{\boldsymbol{c}}$ & 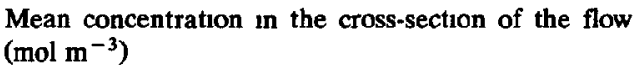 \\
\hline$c_{0}$ & $\begin{array}{l}\text { Concentration in the donor stream at } x=0 \text { for } t>0 \\
\left.(\mathrm{~mol} \mathrm{~m})^{-3}\right)\end{array}$ \\
\hline$c_{\mathrm{dm}}$ & $\begin{array}{l}\text { Concentration at the donor stream/membrane inter- } \\
\text { face }\left(\mathrm{mol} \mathrm{m}^{-3}\right)\end{array}$ \\
\hline$c_{\text {am }}$ & $\begin{array}{l}\text { Concentration at the acceptor stream/membrane in- } \\
\text { terface }\left(\mathrm{mol} \mathrm{m}^{-3}\right)\end{array}$ \\
\hline C & $=c / c_{0}$ Dimensionless concentration \\
\hline$\hat{C}$ & $\begin{array}{l}=\hat{c} / c_{0} \text { Dimensionless mean concentration in the } \\
\text { cross-section of the flow }\end{array}$ \\
\hline $\bar{C}$ & Laplace transform of $C$ or $\hat{C}$ \\
\hline$D$ & Molecular diffusion coefficient $\left(\mathrm{m}^{2} \mathrm{~s}^{-1}\right)$ \\
\hline$D_{\mathrm{L}}$ & Axıal-dıspersıon coefficient $\left(\mathrm{m}^{2} \mathrm{~s}^{-1}\right)$ \\
\hline$D_{\text {md }}$ & $=D_{\mathrm{m}} / D_{\mathrm{d}}$ \\
\hline $\begin{array}{l}W_{\text {ad }} \\
F\end{array}$ & $\begin{array}{l}=D_{\mathrm{a}} / D_{\mathrm{d}} \\
\text { Distribution coefficients }\end{array}$ \\
\hline$L$ & Characteristic length $(\mathrm{m})$ \\
\hline $\boldsymbol{p}$ & Laplace complex variable \\
\hline$P$ & $=\bar{u} L / D_{\mathrm{L}}$ Peclet number \\
\hline$s$ & Input function \\
\hline$t$ & Time (s) \\
\hline $\boldsymbol{u}$ & Linear flow-rate $\left(\mathrm{m} \mathrm{s}^{-1}\right)$ \\
\hline $\bar{u}$ & Mean linear flow-rate $\left(\mathrm{m} \mathrm{s}^{-1}\right)$ \\
\hline $\boldsymbol{U}$ & $=\bar{u}_{\mathrm{a}} / \bar{u}_{\mathrm{d}}$ \\
\hline$x$ & Axıal distance (m) \\
\hline$X$ & $=x / L$ Dimensionless axial distance \\
\hline$y$ & Transverse distance (m) \\
\hline$Y$ & $=y / a_{\mathrm{d}}$ Dimensionless transverse distance \\
\hline
\end{tabular}

Greek letters

$\begin{array}{ll}\beta & =\left(a_{\mathrm{d}} / L\right)^{2} \\ \delta & \text { Half-width of the membrane (m) } \\ \psi & \text { Duration of time-injection (s) } \\ \Psi & =\psi \bar{u}_{\mathrm{d}} / L \text { Dimensionless duration of time-injection } \\ \Delta & =\delta / a_{\mathrm{d}} \text { Dimensionless half-width of the membrane } \\ \theta & =t \bar{u}_{\mathrm{d}} / L \text { Dimensionless time } \\ \tau & =D_{\mathrm{d}} L /\left(\bar{u}_{\mathrm{d}} a_{\mathrm{d}}^{2}\right) \text { Fourier number of the donor stream }\end{array}$

a Subscripts $d$, a and $m$ refer to the donor stream, the acceptor stream and the membrane, respectively, o refers to overall, pulse and step refer to pulse- and stepwise input functions, respectively

are given in Appendix $A$ and the symbols and their definitions are given in Table 1)

$$
\frac{\partial C_{\mathrm{d}}}{\partial \theta}=\tau\left(\beta \frac{\partial^{2} C_{\mathrm{d}}}{\partial X^{2}}+\frac{\partial^{2} C_{\mathrm{d}}}{\partial Y^{2}}\right)-\frac{3}{2} Y(2-Y) \frac{\partial C_{\mathrm{d}}}{\partial X}
$$

$$
\begin{aligned}
\frac{\partial C_{\mathrm{m}}}{\partial \theta}= & D_{\mathrm{md}} \tau\left(\beta \frac{\partial^{2} C_{\mathrm{m}}}{\partial X^{2}}+\frac{\partial^{2} C_{\mathrm{m}}}{\partial Y^{2}}\right) \\
\frac{\partial C_{\mathrm{a}}}{\partial \theta}= & D_{\mathrm{ad}} \tau\left(\beta \frac{\partial^{2} C_{\mathrm{a}}}{\partial X^{2}}+\frac{\partial^{2} C_{\mathrm{a}}}{\partial Y^{2}}\right) \\
& -\frac{3}{2} \frac{U}{A^{2}}\left[-Y^{2}+2(2+A+2 \Delta)\right. \\
& -4(1+\Delta)(1+A+\Delta)] \frac{\partial C_{\mathrm{a}}}{\partial X}
\end{aligned}
$$

The initial and boundary conditions of Eqns 1-3 are

$C_{\mathrm{d}}(0, X, Y)=C_{\mathrm{m}}(0, X, Y)=C_{\mathrm{a}}(0, X, Y)=0$

for $X>0$

$C_{\mathrm{d}}(\theta, 0, Y)=s(\theta)$,

$C_{\mathrm{m}}(\theta, 0, Y)=C_{\mathrm{a}}(\theta, 0, Y)=0$

where

$s(\theta)=\left\{\begin{array}{ll}1 & 0 \text { for } \theta \geqslant 0 \\ 0 & 0 \text { for } \theta<0\end{array}\right.$ for step-function input

and

$s(\theta)= \begin{cases}1 & 0 \text { for } \Psi \geqslant \theta \geqslant 0 \\ 0 & 0 \text { for } 0>\theta \text { or } \theta>\Psi\end{cases}$

for pulse-function input

$C_{\mathrm{d}}(\theta, \infty, Y)=C_{\mathrm{m}}(\theta, \infty, Y)=C_{\mathrm{a}}(\theta, \infty, Y)=0$

$\frac{\partial C_{\mathrm{d}}(\theta, X, 0)}{\partial Y}=0$

$F_{\mathrm{d}} C_{\mathrm{d}}(\theta, X, 2)=C_{\mathrm{m}}(\theta, X, 2)$

$\frac{\partial C_{\mathrm{d}}(\theta, X, 2)}{\partial Y}=D_{\mathrm{md}} \frac{\partial C_{\mathrm{m}}(\theta, X, 2)}{\partial Y}$

$F_{\mathrm{a}} C_{\mathrm{a}}(\theta, X, 2+2 \Delta)=C_{\mathrm{m}}(\theta, X, 2+2 \Delta)$

$D_{\mathrm{ad}} \frac{\partial C_{\mathrm{a}}(\theta, X, 2+2 \Delta)}{\partial Y}=D_{\mathrm{md}} \frac{\partial C_{\mathrm{m}}(\theta, X, 2+2 \Delta)}{\partial Y}$

$\frac{\partial C_{\mathrm{a}}(\theta, X, 2+2 A+2 \Delta)}{\partial Y}=0$

The pulse-function input is identical with the so-called "tıme-injectıon" [20], which can be considered as a realistic approximation to a valve injection [21]

Usually for evaluating the performance of a given dialyser one is interested in the mean con- 
centrations in the cross-sections of the donor and acceptor channels and the membrane, defined as

$\hat{C}(\theta, X)=\frac{1}{Y_{2}-Y_{1}} \int_{Y_{1}}^{Y_{2}} C(\theta, X, Y) \mathrm{d} Y$

where $Y_{1}$ and $Y_{2}$ are the corresponding transverse coordınates ( $F_{1 g} 1$ )

\section{SOLUTION OF THE MODEL}

The model consists of three linear partial differential equations of the second order (Eqns 1-3), each having three independent variables ( $1 \mathrm{e}$, one temporal and two spatial) In a previous paper a fast numerical technique for the solution of such type of problems was proposed [22] As a first step according to this technique the original time-domain equations must be transformed into the Laplace domain

$$
\bar{C}(X, Y)=\int_{0}^{\infty} C(\theta, X, Y) \exp [-p \theta] \mathrm{d} \theta
$$

(the transformed equations are presented in Appendix B)

The transformed boundary conditions are identical with those for Eqns 1-3 provided that the concentrations are replaced with their Laplace transforms, the only exception being the boundary condition of Eqn 1 at $X=0,1 \mathrm{e}$,

$$
\bar{C}(0, Y)=\left\{\begin{array}{l}
1 / p \text { for step-function input } \\
{[1-\exp (-\Psi p)] / p} \\
\text { for pulse-function input }
\end{array}\right.
$$

The Laplace domain equations (Appendix B) were solved for the case of step-function input by the implicit finite-difference method [23] for $p_{t}=$ $2 \iota+1$, where $\imath=0,1, \quad, 15$ The Laplace transform of the mean concentration in the cross-section of both channels and of the membrane (Eqn 4) was calculated at $X=1$ by Simpson's rule [23] The numerical inverse Laplace transformation was performed by the method employing expansion of the Laplace domain function into Fourner sine series $[22,24]$

The solution of Eqns 1-3 for pulse-function input was obtained directly from the step-func- tion solution by means of the following relationship [25]

$$
\hat{C}_{\text {pulse }}(\theta, 1)=\hat{C}_{\text {step }}(\theta, 1)-\hat{C}_{\text {step }}(\theta-\Psi, 1)
$$

For checking the accuracy of the results obtained, Eqns 1-3 were also solved by the much slower implicit alternating-direction finite-difference method [23] for some sets of parameter values Very good agreement between the results obtained by the two numerical methods was obtained

The numerical procedures outlined above were programmed in $\mathrm{C}$ and the corresponding programs were run on IBM/PC compatible and VAX computers

INFLUENCE OF THE MAIN PARAMETERS OF THE DIALYSER ON ITS BEHAVIOUR

The performance of a given dialyser is usually characterized by the amount of solute transferred from the donor to the acceptor stream, which in general is time dependent Under steady-state conditions the effectiveness of the dialyser can easily be quantified by the mean solute concentrations in the cross-sections of both streams ( $1 \mathrm{e}$, $\hat{C}_{\mathrm{d}}$ and $\hat{C}_{\mathrm{a}}$ ) at the entrance and at the exit of the dialyser, respectively Under dynamic conditions (1 e, in flow-injection manifolds), instead of the steady-state concentrations the maximum concentrations at the exit of the dialyser should be used In this study the concentration ratio

$E=\hat{C}_{\mathrm{a}} / \hat{C}_{\mathrm{d}}$

will be used as a quantitative measure of the performance of the dialyser in further considerations The maximum possible value of $E$ corresponding to equilibrium between the flund phases and the membrane is equal to the ratio of the relevant distribution coefficients, $1 \mathrm{e}, F_{\mathrm{d}} / F_{\mathrm{a}}$ Under dynamic conditions the ratio $E$, unlike under steady-state conditions, can be expected to depend also on the sample size expressed as $\Psi$, which is a parameter not directly related to the dialyser For this reason the value of $E$ under steady-state conditions is more informative in characterizing the efficiency of a given dialyser 
Another reason emphasizing the appropriateness of the steady-state $E$ is based on the fact that the dimensionless steady-state concentrations in both channels are interconnected by

$\hat{C}_{\mathrm{d}}+U A \hat{C}_{\mathrm{a}}=10$

which allows the corresponding ratio $E$ (Eqn 8) to be calculated once the value of one concentration is known A simılar simple relationship cannot be derived under dynamic conditions In this connection it seems expedient to investigate the possibility of using the value of $E$ under steadystate conditions to characterize the performance of the same dialyser under dynamic conditions as well

\section{Comparson of $E$ under dynamic and steady- state condittons}

The parameters influencing both the dynamic and the steady-state value of $E$ of a given dialyser are $\tau, A, U, F_{\mathrm{d}}, F_{\mathrm{a}}, D_{\text {md }}$ and $D_{\text {ad }}$ As has been already mentioned, the additional parameter affecting $E$ under dynamic conditions is the sample size expressed as the dimensionless time of injection $\Psi$ In $\mathrm{Fig} 2$ the dependence of $E$ on $\Psi$ is presented for different $\tau$ values and for membrane thickness equal to zero and 01 , respectively As can be seen in the case of zero membrane thıckness and low $\tau$ values $(\tau<07)$, which in the case of two impermeable walls leads to double-humped peaks [21], the sample size is of crucial importance for $E$ This effect is a consequence of the fact that the dispersion pro-

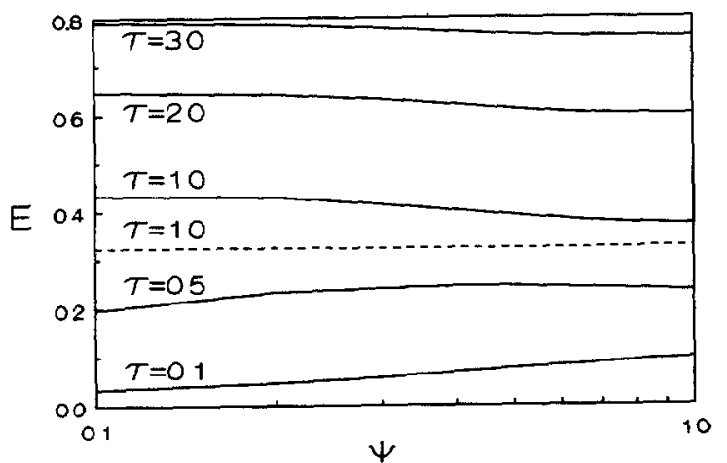

Fig 2 Influence of $\Psi$ on $E$ for different $\tau$ values and for $\Delta \rightarrow 0$ (solid lines) and $\Delta=01$ (dashed line) cess in this instance is dominated by convection (1 e , by the parabolic velocity profile), which leads to considerably higher concentrations in the middle of the donor stream than at its boundaries at one of which is the interface with the acceptor stream This effect decreases with increase in the sample size, thus approaching the steady-state value of $E$

For higher $\tau$ values an opposite effect can be observed, $1 \mathrm{e}$, small samples produce higher $E$ values than larger samples This phenomenon can be explained by the fact that the dispersion process is governed by both convection and diffusion This leads to a high degree of concentration uniformity in the transverse direction in the donor stream, which is favourable for mass transfer However, the loss of solute in the section of the moving sample plug with highest concentration cannot be effectively compensated for by small sample sizes (e g, $\Psi<02)$ This leads to a lower maximum concentration in the donor stream $\left(\hat{C}_{\mathrm{d}}\right)$ and thus to higher $E$ values (Eqn 8) In some instances this may cause even the formation of a double-humped peak in the donor stream (Fig 3) As with low Fourier numbers, by increasing the sample size the $E$ ratio approaches its steady-state value When the dispersion process is dominated by diffusion (1 $\mathrm{e}$, for high $\tau$ values) the sample size hardly affects $E$ When the diffusion within the membrane influences the overall mass transfer ( $\left.F_{1 g} 2, \Delta=01\right)$ the membrane plays the role of a buffer with respect to the effects mentioned above The reason for this is that the concentration gradient at the membrane/donor stream interface is lower than in the case of direct contact between the two flows ( $\mathrm{e}, \Delta \rightarrow 0$ ) For small sample sizes it must be taken into consideration that a portion of the solute is temporarily retained in the membrane, which leads to lower and broader concentrationtıme curves in the acceptor stream In such cases the corresponding $E$ value will be additionally decreased (Fig 2)

In practical parallel-plate dialysers the Fourier numbers of the streams are usually greater than 10 For such $\tau$ values the dynamic $E$ values do not differ from the corresponding steady-state value by more than $10 \%$ even in the most un- 
favourable case of zero membrane thickness In addition, they do not alter the character of the dependence $E$ on the parameters of the dialyser (Fig 4) On the basis of these facts, it can be concluded that the steady-state value of $E$ for a given dialyser can be used as a characteristic of the efficiency of its performance under both steady-state and dynamic conditions

In the following the dependence of the ratio $E$ on the main design and operational parameters of a parallel-plate dialyser and on some of the most important physical properties of the membrane and the flowing fluids for the mass-transfer process will be investigated under steady-state conditions Their effect on the physical phenomena that most influence the overall mass-transfer process ( $1 \mathrm{e}$, the axial and the transverse diffusion and the forced axial convection) will also be elucidated If not stated otherwise the dimensionless parameters of the dialyser $A, U, F_{\mathrm{d}}, F_{\mathrm{a}}, D_{\mathrm{md}}$ and $D_{\text {ad }}$ will be assumed to be equal to unity in the subsequent simulations

\section{Influence of axial diffusion in the flow}

The parameter that has the greatest influence on the axial diffusion in a parallel-plate laminar
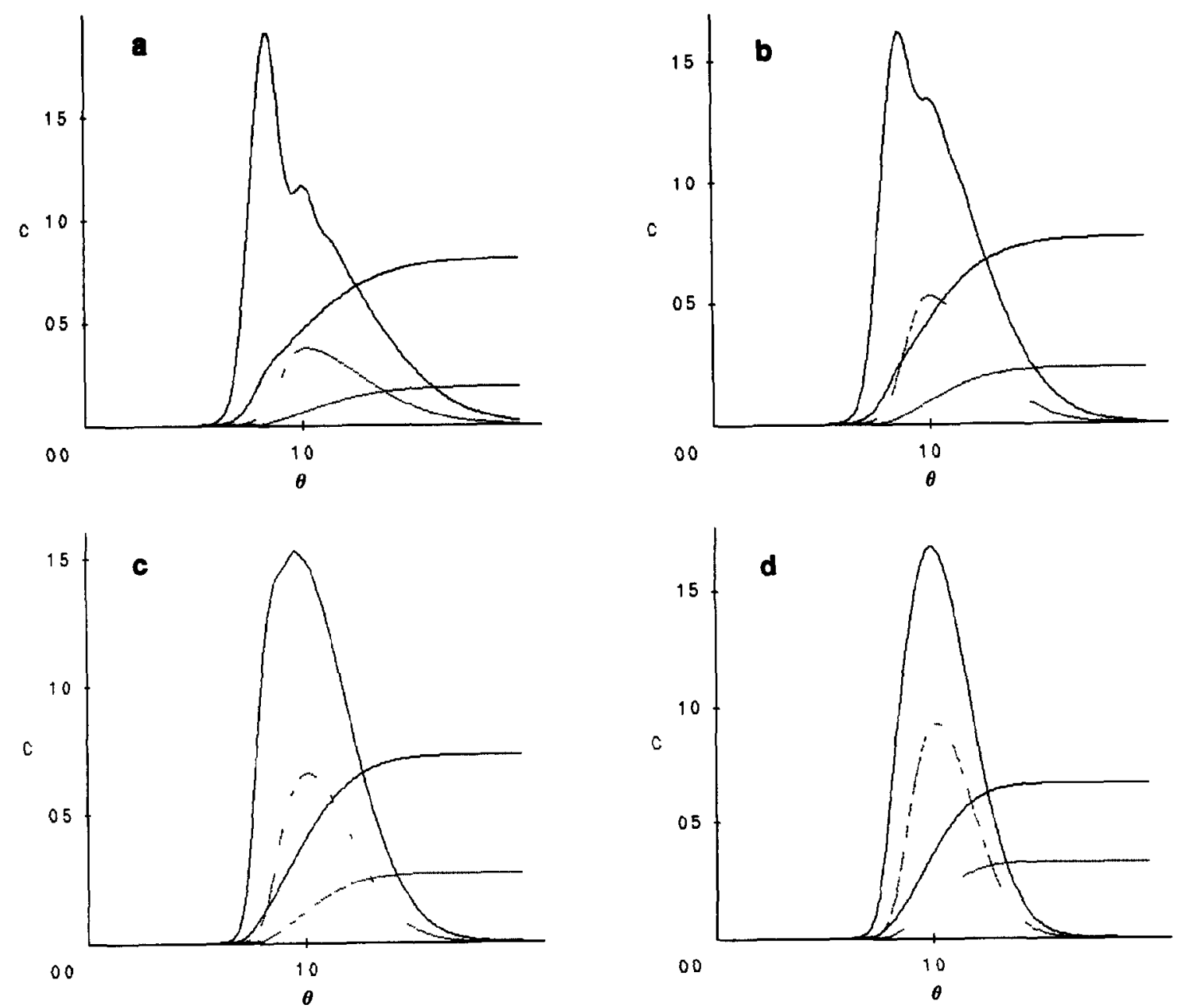

Fig 3 Concentration-time curves in the case of step- and pulse-wise function $(\Psi=01)$ input for $\tau=$ (a) 050 , (b) 075 , (c) 100 and (d) 150 Solid lines, $\hat{C}_{\mathrm{d}}$, dotted lines, $\hat{C}_{\mathrm{a}}$ 


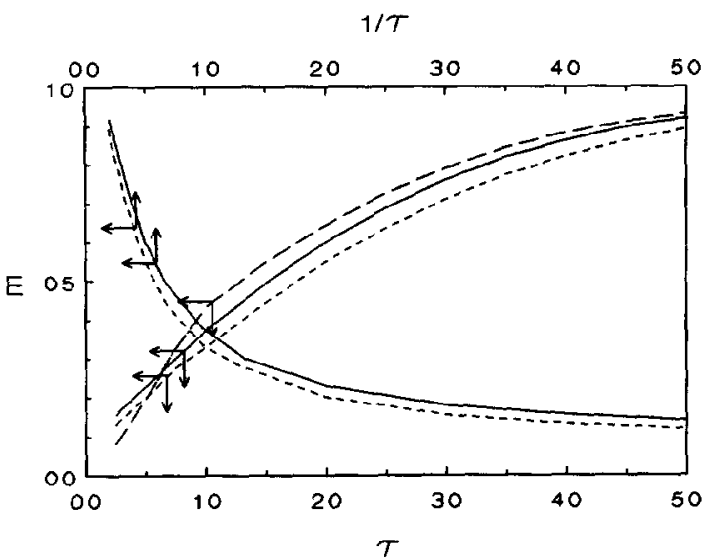

Fig 4 Influence of $\tau$ and $1 / \tau\left(u_{\mathrm{d}} \sim 1 / \tau\right)$ on $E$ (steady-state) for $\Delta \rightarrow 0$ (solid line) and $\Delta=01$ (dashed line), and on $E$ (transient) for $\Delta \rightarrow 0$ and $\Psi=01$ (dot-dashed line)

flow is the geometrical dimensionless group $\beta$, equal to the square of the ratio between half the height of the donor channel and the length of the dialyser Numerous investigations of heat and mass transfer in tubes under various wall boundary conditions showed that the axial conduction or diffusion could affect the overall transfer process only in the entrance region of the test section of the flow system where mass or heat transfer takes places [26-29] For co-current and single-stream parallel-plate flow heat exchange under steady-state conditions, Nunge et al [30] showed that for $16 \times 10^{-3} \tau^{-2}>\beta$ the effect of axial conduction is negligible Similar results were obtained for transient mass transfer in a parallelplate laminar flow system [21] Numerical simulations in the case of an infinitely thin membrane showed that for analytical flow-through dialysers with reasonable $\tau$ and $\beta$ values (1 e, 05-3 0 for $\tau$ and $1 \times 10^{-9}-1 \times 10^{-5}$ for $\beta$ ) the axial diffusion does not affect the overall mass-transfer process For this reason, Eqns 1 and 3 can be simplified by neglecting the axial diffusion terms ( $1 \mathrm{e}$, $\tau \beta \partial^{2} C_{t} / \partial X^{2}$, where $\iota=d, a$ )

\section{Influence of axaal diffusion in the membrane}

In addition to the flowing streams on both sides of the membrane, axial diffusion occurs also inside the membrane itself It may be questioned to what extent this process can affect the overall performance of the dialyser Davis and Gill [31] investigated the effect of axial conduction in the wall in the case of laminar flow under steady-state conditions assuming that the heat flux on the other side of the wall was constant The results obtained for values of the parameters of the system mentioned above valid for practical flowthrough dialysers $\left(1 \mathrm{e}, 2 \beta^{05}<1\right.$ and $\left.\Delta \beta^{05}<1\right)$ showed that outside the entrance region the axial conduction in the wall will not affect the heattransfer process

From Eqn 1 it follows that again the parameters $\beta$ and $\tau$ together with $\Delta$ and $D_{\text {md }}$ characterize mainly the effect of the magnitude of the membrane axial diffusion on the overall masstransfer process Equations 1-3 were solved in the cases of $\tau, \beta$ and $\Delta$ varying in the ranges $10-30,0-1 \times 10^{-4}$ and $005-10$, respectively The mean concentration-tume profiles $[\hat{C}(\theta, 1)]$ at $X=1$ calculated for different $\beta$ values, provided that $\tau$ and $\Delta$ were kept constant, were compared The results obtained showed that even for $\Delta=1$, which is a fairly extreme value for practical parallel-plate dialysers, the concentratıon-tıme curves for $\beta=0$ and $\beta=1 \times 10^{-4}$ differ very slightly from each other For more realistic values of $\Delta$ a total overlap of the corresponding curves was observed Similar effects were found when $D_{\mathrm{md}}$ was varied within one order of magnitude All these results show unambiguously that the axial diffusion in the membrane can be neglected, $1 \mathrm{e}, \tau \beta \partial^{2} C_{m} / \partial X^{2}=0$, thus additionally simplifying the mathematical description of the parallel-plate dialyser considered in this study

\section{Influence of transverse diffusion}

The parameters determining the influence of the transverse diffusion are the molecular diffusion coefficients of the solute in the flowing fluids and in the membrane In Eqns 2 and 3 the features of both the membrane and the acceptor stream are related to those of the donor stream so that the dimensionless groups $D_{\text {md }}$ and $D_{\text {ad }}$ can be considered as the relatıve diffusion coefficients in the membrane and the acceptor stream, respectrvely The value of this parameter for the donor stream is unity by definition 
Another parameter of the dialyser that considerably affects the mass-transfer process, which is also dependent on the molecular diffusion coefficient, is the Fourier number ( $\tau$ ), related again to the donor stream By simulations, the influence of the various parameters was studied It was found that in the case of a stepwise input function by increasing the $\tau$ value the concentrationtime curves in both channels at $X=10$ become

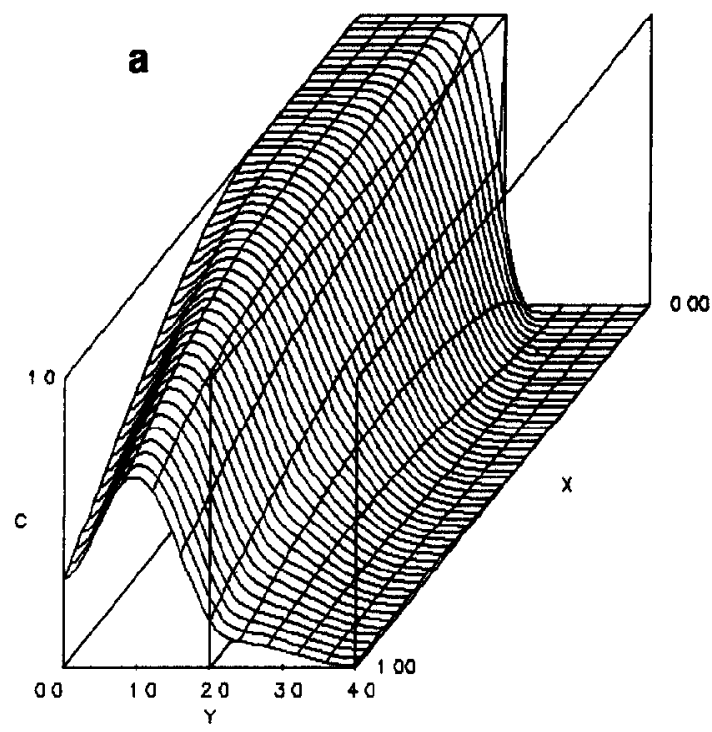

more symmetrical with respect to their inflection point, which approaches $\theta=10$ (Fig 3) In the transverse direction the symmetry with respect to the central plane in each channel also increases at high Fourier numbers ( $\mathrm{F} 1 \mathrm{~g}$ ) As can be expected, by increasing the value of this parameter $E$ will also become greater Figure 4 illustrates the steady-state $\tau$ dependence of $E$ The membrane thickness does not change the charac-

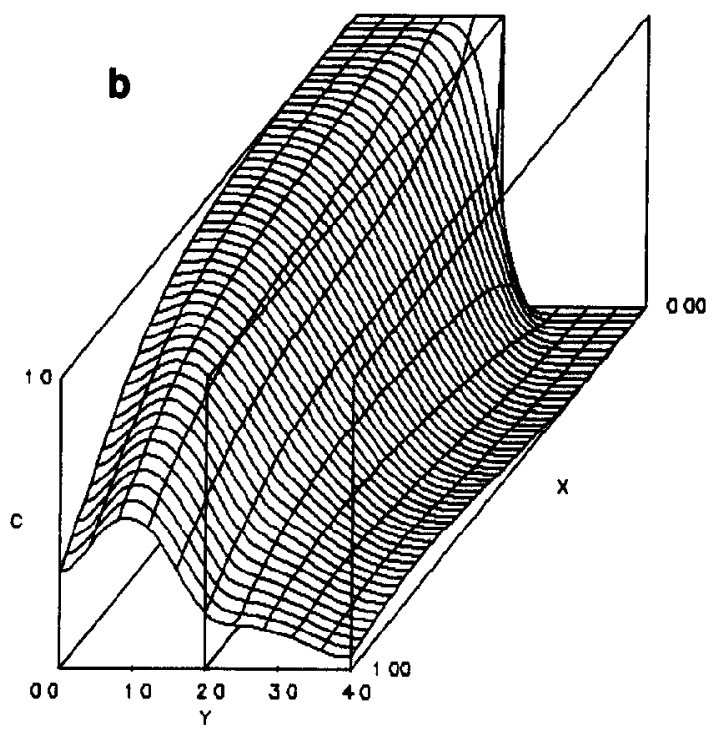

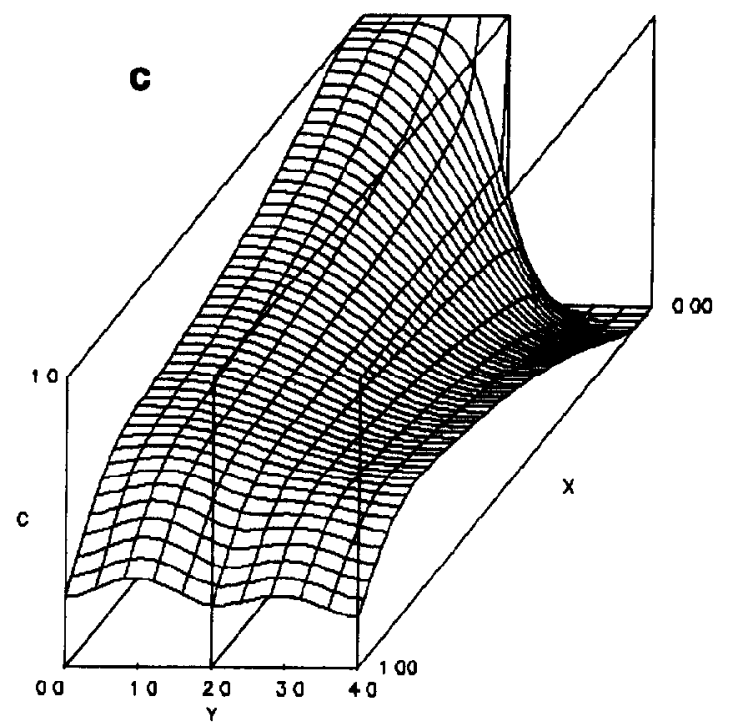

Fig 5 Spatial distribution of the solute in the flow system at $\theta=10$ if $\Delta \rightarrow 0$ for $\tau=$ (a) 050 , (b) 100 and (c) 400 
ter of this dependence From Fig 4 it can be seen that for high Fourier numbers $(\mathrm{eg}, \tau>45)$ the degree of transfer of the dialyser increases very slowly with mcreasing $\tau$ and it asymptotically approaches unity at $\tau \rightarrow \infty$ Takıng into consideration that the Fourier number is linearly proportional to the mean residence time, it becomes evident that for analytical flow-through mantfolds, where the sample throughput rate should be high, an excessive increase in $\tau$ is undesirable for improving the performance of their dialysers Alternative solutions based on manipulation of the other parameters of the dialyser should be found

The performance of the dialyser when the reduced diffusion coefficients in the membrane and the acceptor stream differ from unity are illustrated in Fig 6 Because the diffusion coefficient in the membrane is usually lower than that in the fluid, only values of $D_{\text {mo }}$ lower than unity are considered From Fig 6 it can be seen that the performance of the dialyser does not depend substantially on the properties of the membrane in the Fourier number range 10-30 if $D_{\text {md }}>02$ This result means that in the case of porous media where $D_{\text {md }}=02-06[11]$, the corresponding membranes will have little effect on the transfer process However, membranes with $D_{\mathrm{md}}<02$ influence considerably the performance of the dialyser (Fig 6) The reduced diffusion coefficient of the acceptor stream $\left(D_{\mathrm{ad}}\right)$ was varied in

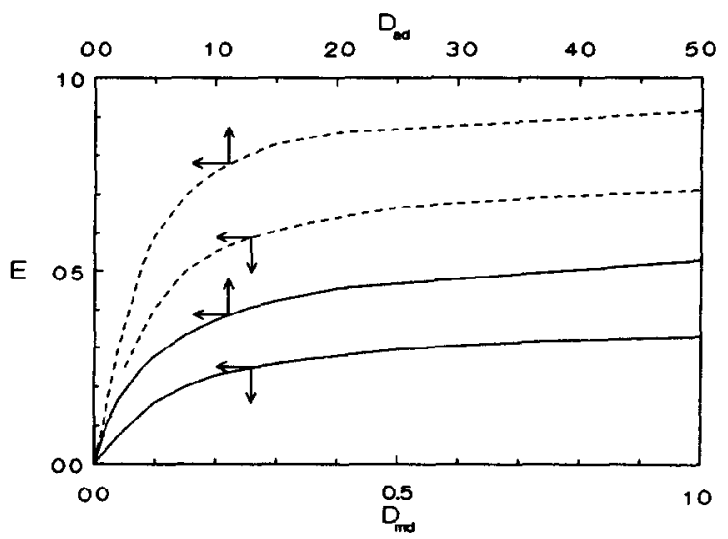

Fig 6 Dependence of $E$ on $D_{\text {md }}$ and $D_{\text {ad }}$ for $\tau=10$ (solid lines) and $\tau=30$ (dashed lines)

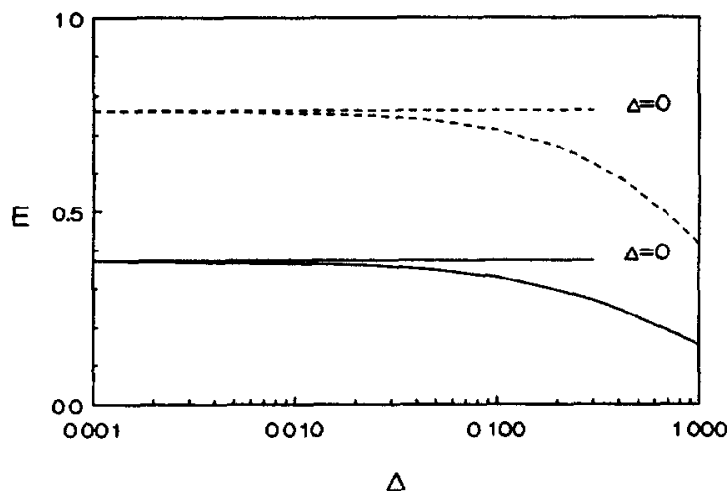

Fig 7 Effect of the membrane thickness $(\Delta)$ on $E$ for $\tau=10$ (solıd lines) and $\tau=30$ (dashed lines)

the range 0-5 The results obtained (Fig 6) show that $E$ is sharply reduced if the diffusion coefficient in the acceptor stream is lower than that in the donor stream whereas the reverse situation does not lead to a substantial improvement in the performance of the dialyser The Fourier number does not affect qualitatively the relationship described above (Fig 6)

\section{Influence of geometry of the dialyser}

The geometrical parameters of the dialyser are the dimensionless half-thickness of the membrane $(\Delta)$ and the dimensionless half-height of the acceptor channel $(A)$, the length of the dialyser $(L)$, the half-height of the donor channel $\left(a_{\mathrm{d}}\right)$ and the geometrical dimensionless group $\beta=\left(a_{\mathrm{d}} / L\right)^{2}$ It was already shown that the parameter $\beta$ does not affect the mass-transfer process in the range of parameter values considered in this study

The influence of the membrane thickness on the concentration ratio $E$ is illustrated in Fig 7 It can be seen that for $\Delta<001$ the assumption of an infinitely thin membrane $(1 \mathrm{e}, \Delta \rightarrow 0)$ will lead to a relative error not greater than $25 \%$, provided that $\tau>10$ It should be taken into consideration that if instead of $E$ only $\hat{C}_{\mathrm{d}}$ or $\hat{C}_{\mathrm{a}}$ is of interest, then it can be expected that the assumption $\Delta \rightarrow 0$ can also be used for higher $\Delta$ values The error in calculating $\hat{C}_{\mathrm{a}}$ as a function of $\Delta$, if the assumption $\Delta \rightarrow 0$ was used, was investigated for different $\tau$ values $(\tau=10-30)$ Almost linear 


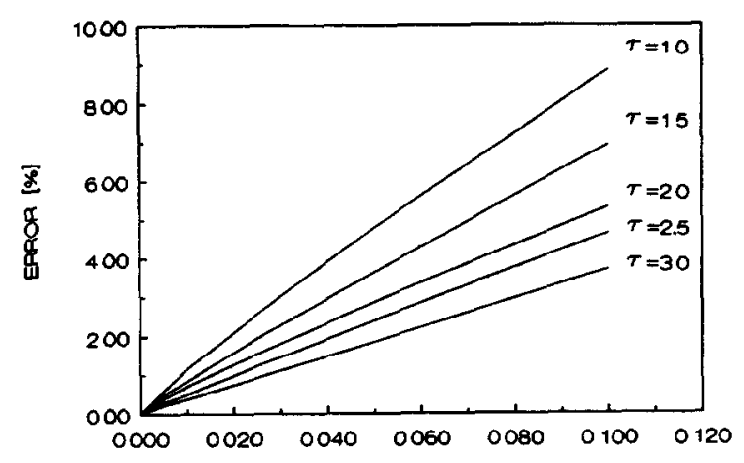

$\Delta$

Fig 8 Relative error in calculating $\hat{C}_{\mathrm{a}}$ assuming that $\Delta \rightarrow 0$ versus the actual membrane thickness for different Fourier numbers

relationships were obtained in the range of $\Delta$ values from 0.0 to 01 (Fig 8) It can be seen that for higher $\tau$ values the transfer process within the membrane can be neglected for relatively thick membranes, thus coverung numerous pract1cal cases

Another geometrical parameter playing an umportant role in the performance of parallel-plate dialysers is the ratio between the heights of the acceptor and the donor channels $(A)$ The dependence of the degree of transfer on $A$ for two different $\tau$ values ( $\mathrm{e}, 10$ and 30 ) is illustrated in Fig 9 As can be expected, on decreasing the parameter $A$ the degree of transfer strongly increases This fact offers a promising approach for improving the sensitivity of detection in flow-in-

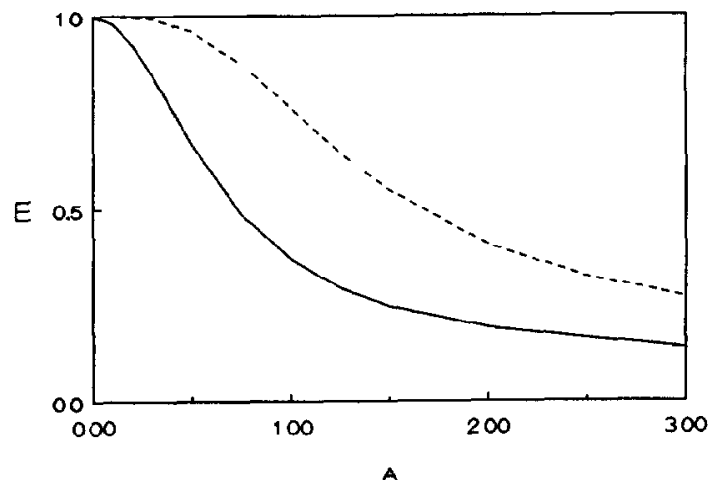

Fig 9 Influence of $A$ on $E$ for $\tau=10$ (solid line) and $\tau=30$ (dashed line) jection manifolds with dialysis separation However, the value of $A$ cannot be decreased without limitations To prevent deformations of the membrane the flow-rates in both channels must be kept close to each other This means that by decreasing the ratio $A$ the Fourier number of the donor stream will sharply decrease, $1 \mathrm{e}, \tau_{\mathrm{a}}=\tau_{\mathrm{d}} A^{2}$ This may lead to a reduced reproducibility and precision under flow-injection conditions, manly owing to the formation of double-humped peaks It can be calculated (Eqns 8 and 9) that by increasing $A$ the decrease in $E$ (Fig 9), caused by the dilution effect in the acceptor stream, is accompanied by a decrease in the concentration in the donor stream For this reason, $A$ values greater than unity could be recommended for improving the performance of haemodialysers because in these mass-exchange devices one is arming at a low concentration in the donor stream rather than a high concentration in the acceptor stream

The Fourier number is directly proportional to the length of the dialyser $(L)$ and inversely proportional to the square of the half-height of the donor channel For this reason the $\tau$ dependence of $E$ in Fig 4 also illustrates the influence of $L$ on the performance of the dialyser The dependence of the ratio $E$ on $a_{\mathrm{d}}\left(a_{\mathrm{d}} \sim \tau^{-1 / 2}\right)$ is illustrated in Fig 10 The values of $E$ for high Fourier numbers were extrapolated From Fig 10 it follows that a decrease in the height of the channels

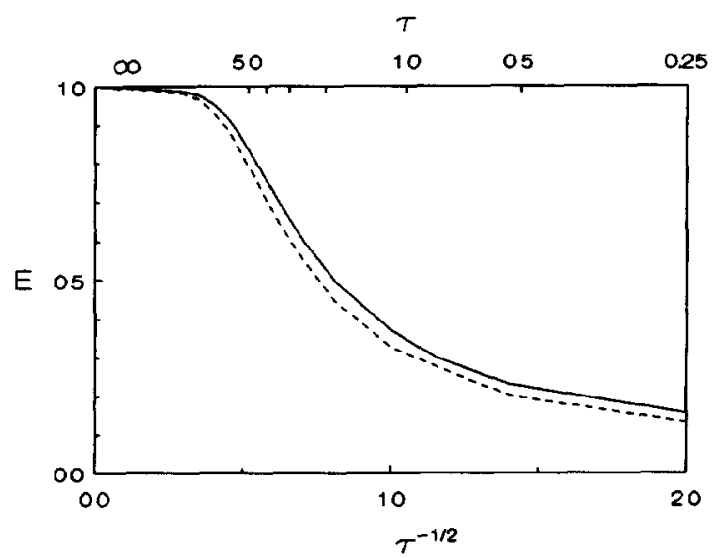

Fig 10 Dependence of $E$ on the height of the donor channel $\left(a_{\mathrm{d}} \sim \tau^{-1 / 2}\right.$ ) $\Delta \rightarrow 0$ (solid line) and $\Delta=01$ (dashed line) 
In the $\tau$ range $10-50$ can considerably improve the performance of the dialyser $A$ membrane with a moderate width does not change the character of this dependence

\section{Influence of flow-rate}

The effect of the flow-rates of the two streams on the mass transfer in the dialyser considered in this study can be expressed by the Fourier number $(\tau)$ and the reduced flow-rate of the acceptor stream $(U)$

The Fourier number is inversely proportional to the flow-rate and for this reason its influence can be illustrated as the $1 / \tau$ dependence of $E$ (Fig 4) If instead of $E$ the steady-state concentration in the acceptor stream $\left(\hat{C}_{\mathrm{a}}\right)$ is plotted against $1 / \tau$, a similar relationship to that given by Bernhardsson et al [11] and Van Staden and Van Rensburg [32] can be observed

The influence of the flow-rate ratio $(U)$ in the range $05-20$ on the performance of the dialyser for two different Fourier numbers $(\tau=10$ and $30)$ and for $\Delta \rightarrow 0$ and $\Delta=01$ is illustrated in Fig 11 As can be expected, by decreasing the flow-rate in the acceptor channel, thus making $U$ less than 1, the ratio $E$ is increased However, as was pointed out earlier, if $U$ is different from unity a pressure difference between the two channels of the dialyser considered in this study will be created As a result, the membrane could be severely deformed and damaged, which will

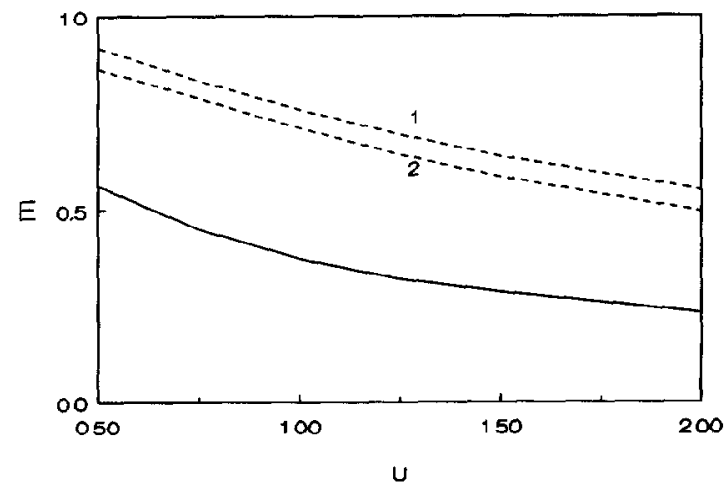

Fig 11 Influence of $U$ on $E$ for $\tau=10$ (solid line) and $\tau=30$ (dashed lines) Curves 1 and 2 refer to $\Delta \rightarrow 0$ and $\Delta=01$, respectively

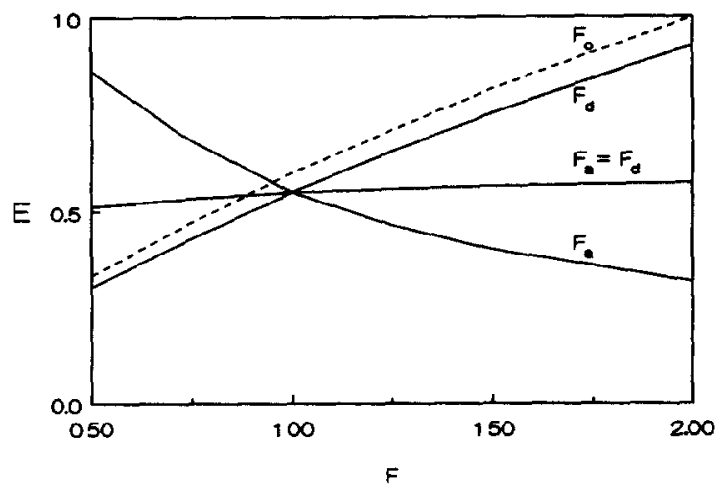

Fig 12 Dependence of $E(\tau=20)$ on the distribution coefficients in the case of $\Delta=01$ (solid lines) and $F_{\mathrm{o}}=F_{\mathrm{d}} / F_{\mathrm{a}}$ for $\Delta \rightarrow 0$ (dashed line)

inevitably lead to malfunctioning of the dialyser If the dialyser is part of a flow manifold, then to prevent the undesirable effect mentioned above one must take into consideration the hydraulic resistances in all of the flow-through sections and not only in the dialyser

\section{Influence of distribution coefficients}

The ratio $E$ can be considerably affected by the distribution coefficients of the solute for the membrane and the fluids in both channels The influence of $F_{\mathrm{d}}$ and $F_{\mathrm{a}}$ on $E$ is shown in Fig 12 As can be expected, they have an opposite effect on the performance of the dialyser $A$ realistic practical case is when the solvent on both sides of the membrane is the same so that $F_{\mathrm{d}}=F_{\mathrm{a}}$ As can be seen from Fig 12, in such a case the masstransfer remains almost independent of the distrbution coefficients Simulations performed for the case where the solvents in both channels are different and $F_{\mathrm{a}} / F_{\mathrm{d}} \neq 1$ showed that the ratio $E$ can be increased considerably In the case of very thin membranes $(\Delta \rightarrow 0)$ the two streams can be regarded as directly contacting with each other The distribution coefficient in this case is defined as $F_{\mathrm{o}}=F_{\mathrm{d}} / F_{\mathrm{a}}$ and its effect on $E$ is similar to that of $F_{\mathrm{d}}$ (Fig 12) The above considerations show that only by an appropriate choice of the solvent in the acceptor stream can the performance of the dialyser be improved considerably 


\section{Conclusions}

The mass transfer in a parallel-plate dialyser with co-current fully developed laminar flow in both channels was described mathematically using the convective-diffusion equation for parallel-plate laminar flow and Fick's second law The efficiency of the dialyser was characterized by the concentration ratio $(E)$ at its outlet in the acceptor and the donor streams It was found that the steady-state value of $E$ can also be used for evaluating the efficiency of the dialyser under dynamic conditions (e g, flow-injection analysis) as well By solving the equations mentıoned above for different values of the main parameters of the dialyser and of the physical constants which are the most relevant for the mass-transfer process, important conclusions for simplifying the mathematical description and for optımızing the performance of the dialyser were drawn They could be summarized as follows

Possibilities for simplifying the mathemattcal description First, the axial diffusion in both streams and in the membrane is insignificant for the overall mass-transfer process and the axial diffusion terms in the corresponding partial differential equations can be neglected Second, for relatively thin membranes the membrane can be assumed to be infinitely thin, which reduces the number of equations constituting the mathematical description of the dialyser by one

Guldelines for optmizing the performance of the dialyser By increasing the Fourier number up to 45 , preferably by reducing the heights of both channels rather than by increasing the mean residence time, which will decrease the sample throughput, the mass-transfer can be substantially increased By the appropriate selection of the carrier solutions with respect to the distribution coefficients of the solute, the concentration ratıo $E$ can be substantially increased By manipulating within certain limits the ratio between the heights of the two channels $(A)$ the performance of the dialyser can be considerably improved In flow-injection dialysers $\boldsymbol{A}$ should not exceed unity whereas in haemodialysers the opposite condition will be more advantageous For membranes of moderate relative thıckness the ratıo $E$ does not depend substantially on the membrane diffusion coefficient, which makes the selection of an appropriate dialysis membrane easier

\section{APPENDIX A}

Mathematical descrotion of a parallel-plate dialyser with co-current laminar flow in both channels

$\frac{\partial c_{\mathrm{d}}}{\partial t}=D_{\mathrm{d}}\left(\frac{\partial^{2} c_{\mathrm{d}}}{\partial x^{2}}+\frac{\partial^{2} c_{\mathrm{d}}}{\partial y^{2}}\right)-\frac{3}{2} u_{\mathrm{d}} \frac{\partial c_{\mathrm{d}}}{\partial x}$
$\frac{\partial c_{\mathrm{m}}}{\partial t}=D_{\mathrm{m}}\left(\frac{\partial^{2} c_{\mathrm{m}}}{\partial x^{2}}+\frac{\partial^{2} c_{\mathrm{m}}}{\partial y^{2}}\right)$
$\frac{\partial c_{\mathrm{a}}}{\partial t}=D_{\mathrm{a}}\left(\frac{\partial^{2} c_{\mathrm{a}}}{\partial x^{2}}+\frac{\partial^{2} c_{\mathrm{a}}}{\partial y^{2}}\right)-\frac{3}{2} u_{\mathrm{a}} \frac{\partial c_{\mathrm{a}}}{\partial x}$

where

$$
\begin{aligned}
u_{\mathrm{d}}= & \bar{u}_{\mathrm{d}} \frac{y}{a_{\mathrm{d}}}\left(2-\frac{y}{a_{\mathrm{d}}}\right) \\
u_{\mathrm{a}}= & \bar{u}_{\mathrm{a}}\left[-y^{2}+2\left(2 a_{\mathrm{d}}+2 \delta+a_{\mathrm{a}}\right) y\right. \\
& \left.-4\left(a_{\mathrm{d}}+\delta\right)\left(a_{\mathrm{d}}+a_{\mathrm{a}}+\delta\right)\right] / a_{\mathrm{a}}^{2}
\end{aligned}
$$

The initial and boundary conditions are

$c_{\mathrm{d}}(0, x, y)=c_{\mathrm{m}}(0, x, y)=c_{\mathrm{a}}(0, x, y)=0$

for $x>0$

$c_{\mathrm{d}}(t, 0, y)=c_{0} s(t)$,

$c_{\mathrm{m}}(t, 0, y)=c_{\mathrm{a}}(t, 0, y)=0$

where

$s(t)=\left\{\begin{array}{ll}1 & 0 \text { for } t \geqslant 0 \\ 0 & 0 \text { for } t<0\end{array}\right.$ for step-function input

and

$s(t)= \begin{cases}1 & 0 \text { for } \psi \geqslant t \geqslant 0 \\ 0 & 0 \text { for } 0>t \text { or } t>\psi\end{cases}$

$c_{\mathrm{d}}(t, \infty, y)=c_{\mathrm{m}}(t, \infty, y)=c_{\mathrm{a}}(t, \infty, y)=0$

$\frac{\partial c_{\mathrm{d}}(t, x, 0)}{\partial y}=0$

$F_{\mathrm{d}} c_{\mathrm{d}}\left(t, x, 2 a_{\mathrm{d}}\right)=c_{\mathrm{m}}\left(t, x, 2 a_{\mathrm{d}}\right)$ 


$$
\begin{aligned}
& D_{\mathrm{d}} \frac{\partial c_{\mathrm{d}}\left(t, x, 2 a_{\mathrm{d}}\right)}{\partial y}=D_{\mathrm{m}} \frac{\partial c_{\mathrm{m}}\left(t, x, 2 a_{\mathrm{d}}\right)}{\partial y} \\
& F_{\mathrm{a}} c_{\mathrm{a}}\left(t, x, 2 a_{\mathrm{d}}+2 \delta\right)=c_{\mathrm{m}}\left(t, x, 2 a_{\mathrm{d}}+2 \delta\right) \\
& D_{\mathrm{a}} \frac{\partial c_{\mathrm{a}}\left(t, x, 2 a_{\mathrm{d}}+2 \delta\right)}{\partial y}=D_{\mathrm{m}} \frac{\partial c_{\mathrm{m}}\left(t, x, 2 a_{\mathrm{d}}+2 \delta\right)}{\partial y} \\
& \frac{\partial c_{\mathrm{a}}\left(t, x, 2 a_{\mathrm{d}}+2 a_{\mathrm{a}}+2 \delta\right)}{\partial y}=0
\end{aligned}
$$

\section{APPENDIX B}

Laplace transforms of Eqns 1-3

$$
\begin{aligned}
& \tau\left(\beta \frac{\partial^{2} \bar{C}_{\mathrm{d}}}{\partial X^{2}}+\frac{\partial^{2} \bar{C}_{d}}{\partial Y^{2}}\right)-\frac{3}{2} Y(2-Y) \frac{\partial \bar{C}_{\mathrm{d}}}{\partial X}-p \bar{C}_{\mathrm{d}}=0 \\
& D_{\mathrm{md}} \tau\left(\beta \frac{\partial^{2} \bar{C}_{\mathrm{m}}}{\partial X^{2}}+\frac{\partial^{2} \bar{C}_{\mathrm{m}}}{\partial^{2} Y}\right)-p \bar{C}_{\mathrm{m}}=0 \\
& D_{\mathrm{ad}} \tau\left(\beta \frac{\partial^{2} \bar{C}_{\mathrm{a}}}{\partial X^{2}}+\frac{\partial^{2} \bar{C}_{\mathrm{a}}}{\partial Y^{2}}\right) \\
& \quad-\frac{3}{2} \frac{U}{A^{2}}\left[-Y^{2}+2(2+A+2 \Delta)\right. \\
& \quad-4(1+\Delta)(1+A+\Delta)] \frac{\partial \bar{C}_{\mathrm{a}}}{\partial X}-p \bar{C}_{\mathrm{a}}=0
\end{aligned}
$$

\section{REFERENCES}

1 R B Bird, W E Stewart and EN Lightfoot, Transport Phenomena, Wiley, New York, 1960

2 L Grımsrud and A L Babb, AIChE Symp Ser , 62 (1966) 20,66

3 C K Colton, K A Smith, P Strove and EW Merrill, AIChE J, 17 (1971) 773

4 J M Koolyman, Chem Eng Sc1, 28 (1973) 1149

5 D O Cooney, S-S Kım and E J Davis, Chem Eng Sci, 29 (1974) 173
6 D O Cooney, E J Davis and S -S Kım, Chem Eng J, 8 (1974) 213

7 L T Skeggs, Jr, Am J Clin Pathol, 28 (1957) 311

8 A.H Kadısh and D A Hall, Clin Chem, 11c (1965) 869

9 E H Hansen and J Ruzıcka, Anal Chım Acta, 87 (1976) 353

10 WE van der Linden, Anal Chım Acta, 151 (1983) 359

11 B Bernhardsson, $E$ Martıns and $G$ Johansson, Anal Chım Acta, 167 (1985) 111

12 L Risinger, G Johansson and $\mathrm{T}$ Thorneman, Anal Chm Acta, 224 (1989) 13

13 D R Reneau, Jr, D F Bruley and M H Knısly, AIChE J, 15 (1969) 916

14 E J Davis, D O Cooney and R Chang, Chem Eng J, 7 (1974) 213

15 P F Tomlan and J L Hudson, Int J Heat Mass Transfer, 11 (1968) 1253

16 E M Sparrow and FN de Fanas, Int $J$ Heat Mass Transfer, 11 (1968) 837

17 M Perlmutter and R Siegel, J Heat Transfer, 83 (1961) 432

18 R Siegel and M Perlmutter, J Heat Transfer, 85 (1963) 358

$19 \mathrm{~J} \mathrm{~L} \mathrm{Hudson} \mathrm{and} \mathrm{S} \mathrm{G} \mathrm{Bankoff,} \mathrm{Int} \mathrm{J} \mathrm{Heat} \mathrm{Mass} \mathrm{Transfer,}$ $6(1963) 607$

$20 \mathrm{~J} M$ Rejun, WE van der Linden and $H$ Poppe, Anal Chim Acta, 114 (1980) 105

21 S D Kolev and WE van der Linden, Anal Chım Acta, 247 (1991) 51

22 S D Kolev and WE van der Linden, Num Heat Transfer, in press

23 B Carnahan, H A Luther and J O Wilkes, Applied Numerical Methods, Wiley, New York, 1969

24 S D Kolev and E Pungor, Anal Chım Acta, 194 (1987) 61

25 S D Kolev, Anal Chım Acta, 229 (1990) 183

26 C-J Hsu, AIChE J, 17 (1971) 732

$27 \mathrm{~F}$ Sobron, L Rabanillo and J M Lopez, Int Chem Eng, 30 (1990) 236

28 E Papotsakıs, D Ramkrıshna and H C Lim, AIChE J, 26 (1980) 779

$29 \mathrm{CW}$ Tan and C-J Hsu, Int J Heat Mass Transfer, 15 (1972) 2187

30 R J Nunge, EW Porta and W N Gill, Chem Eng Symp Ser , 63 (1967) 80

31 E J Davis and W N Gill, Int J Heat Mass Transfer, 13 (1970) 459

32 J F van Staden and A van Rensburg, Analyst, 115 (1990) 1049 